\title{
Determining and Labeling the Preference Dimensions of Spatial Audio Replay
}

\author{
Jon Francombe ${ }^{1}$, Tim Brookes, \\ and Russell Mason \\ Institute of Sound Recording \\ University of Surrey \\ Guildford, Surrey, GU2 7XH \\ ${ }^{1}$ Email: j.francombeesurrey.ac.uk
}

\author{
James Woodcock \\ Acoustic Research Centre \\ University of Salford \\ Salford, M5 4WT
}

\begin{abstract}
There are currently many spatial audio reproduction systems in domestic use (e.g. mono, stereo, surround sound, sound bars, and headphones). In an experiment, pairwise preference magnitude ratings for a range of such systems were collected from trained and untrained listeners. The ratings were analysed using internal preference mapping to: (i) uncover the principal perceptual dimensions of listener preference; (ii) label the dimensions based on important perceptual attributes; and (iii) observe differences between trained and untrained listeners. To aid with labelling the dimensions, perceptual attributes were elicited alongside the preference ratings and were analysed by: (i) considering a metric derived from the frequency of use of each attribute and the magnitude of the related preference judgements; and (ii) observing attribute use for comparisons between specific methods. The first preference dimension accounted for over $90 \%$ of the variance in ratings; all participants exhibited a preference for reproduction methods that were positively correlated with the first dimension (most notably 5-, 9-, and 22-channel surround sound). This dimension was related to multiple important attributes, including those associated with spatial capability and absence of distortions. The second dimension accounted for only a very small proportion of the variance, and appeared to separate the headphone method from the other methods. The trained and untrained listeners generally showed opposite preferences in the second dimension, suggesting that trained listeners have a higher preference for headphone reproduction than untrained listeners.
\end{abstract}

\section{INTRODUCTION}

Spatial audio reproduction attempts to achieve some balance between accurately representing a real sound field (in terms of its spatial and timbral properties, e.g. source positions and frequency content), and producing an enjoyable, immersive, and plausible listening experience [1]. Channelbased reproduction has developed over a number of decades in order to better achieve these goals. Two-channel stereo is capable of producing phantom images within the angle subtended by the loudspeakers ( \pm 30 degrees). Five-channel surround sound [2] includes a center loudspeaker to anchor the image for off-center listeners, as well as adding to spatial impression by including loudspeakers behind the listening position. More recently, advanced channel-based systems have been standardised [3], including loudspeakers at different heights. Alongside developing channel-based methods, scene-

This work was supported by the EPSRC Programme Grant S3A: Future Spatial Audio for an Immersive Listener Experience at Home (EP/L000539/1) and the BBC as part of the BBC Audio Research Partnership. Details about the data underlying this work, along with the terms for data access, are available from http://dx.doi.org/10.15126/surreydata.00810078. and object-based reproduction - which remove the dependency on strictly controlled loudspeaker numbers and positionsare also becoming more common [4]. In order to optimise quality of listening experience for end-users, it is important to determine what reproduction methods listeners prefer, as well as the important perceptual attributes that lead to these preference judgements. Perceptual evaluation is necessary for understanding the quality of a spatial audio reproduction system from a listener-centric perspective [5].

A preference rating experiment was performed in which paired comparison preference magnitude judgements were made for eight reproduction methods (headphones with a mixture of stereo and binaural presentation, low-quality mono, mono, stereo, 5-channel, 9-channel, 22-channel, and ambisonic cuboid) and seven programme material items (brass quintet, jazz quintet, pop track, big band, football broadcast, experimental music, and film excerpt) [6]. The excerpts were perceptually loudness matched in a method-of-adjustment experiment [7]. The experiment was conducted in a room with dimensions of $7.85 \mathrm{~m} \times 12.38 \mathrm{~m}$ (with a heavy curtain at $8.23 \mathrm{~m}$ ) $\times 4.00 \mathrm{~m}$. The RT60 was $215 \mathrm{~ms}$ between $0.5 \mathrm{kHz}$ and $2 \mathrm{kHz}$. In the experiment, participants were presented with every possible combination of stimuli (one pair at a time) and asked to indicate on a continuous scale which of the pair they preferred. The experiment was performed by seven trained listeners (undergraduate students in music and sound recording who had all completed a module in technical ear training) and eight untrained listeners (university students or recent graduates from various backgrounds, with no formal technical or musical training at degree level). A text box was provided alongside each paired comparison (see Figure 1); participants were instructed to type the reasons for their preference choice. The responses were clustered using a textclustering algorithm; the clusters were used as the input to a group discussion (performed by the same participants), during which two attribute sets (one for each listener group) were produced [8]. Overlap between the two sets was determined in a follow-up task. A mapping was performed to relate the recorded text responses to the attributes to which they ultimately contributed; these results were used in the attribute score analysis described in Section II-A.

In this paper, the results from the experiment described above are further analysed. The aims of the work were to:

1) objectively assess the perceptual importance of the elicited attributes; 


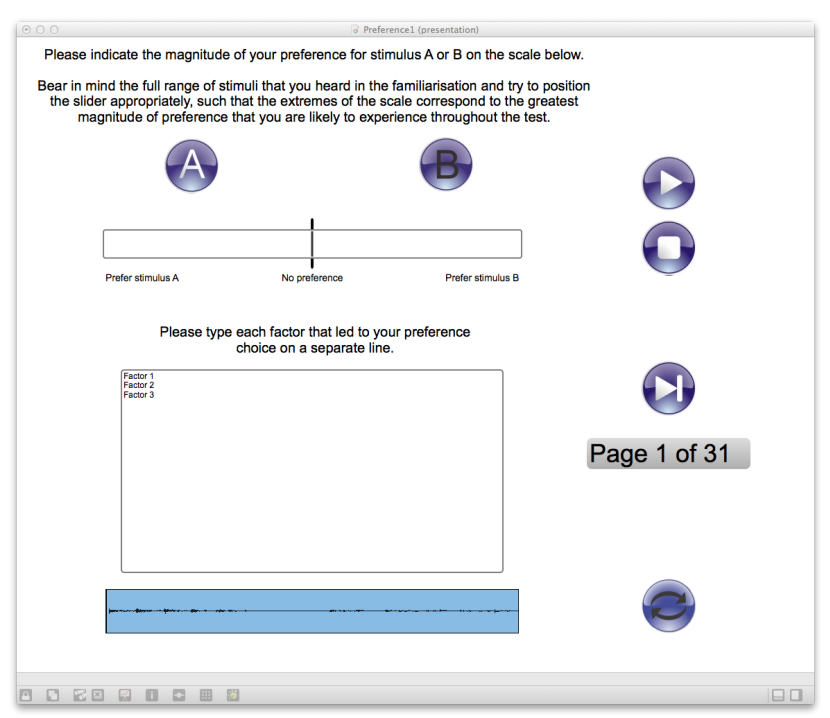

Fig. 1: User interface for the preference rating and free elicitation test

2) determine and label the principal perceptual dimensions of listener preference for spatial audio reproduction methods; and

3) observe differences between trained and untrained listeners.

In Section II, the elicited attribute set is analysed based on overall frequency of attribute use, and further broken down for particular combinations of reproduction methods. Internal preference mapping was used to position the reproduction methods in a perceptual space and determine how this related to listener preference, and the attributes were used to label the resultant dimensions; this process is described in Section III. The results are discussed and summarised in Section IV.

\section{ANALYSIS OF ATTRIBUTES}

As detailed in Section I, a set of perceptual attributes that listeners use for making preference judgements for different spatial audio reproduction methods was elicited. The task that participants were requested to perform was intended to ensure that the elicited attributes all contribute to preference; however, it is likely that some attributes are more important than others. The 'attribute score' metric was developed as a way of quantifying the importance of the attributes; this is described in Section II-A. The dimensional analysis reported in Section III shows where the various reproduction methods fall along preference dimensions. In order to aid the labelling of these dimensions with pertinent perceptual attributes, it is beneficial to look at the attribute use for particular combinations of reproduction methods. However, there are a large number of comparisons, and it is difficult to visualise this data in a concise manner. A method for analysing these comparisons is described in Section II-B.

\section{A. Attribute score}

The attribute score attempts to quantify the perceptual importance of each attribute by considering the frequency with which it was used ${ }^{1}$, as well as the size of the preference judgements alongside which it was used. An attribute might be considered to be perceptually important if was used to describe differences between many stimulus combinations; however, an attribute might also be important if it was used less often but always alongside preference judgements of a high magnitude.

The attribute score $A$ for the $n$th attribute is given by

$$
A_{n}=\left[\sum_{i=1}^{I} f_{n_{i}} \cdot p_{i}\right] \cdot \frac{\max \boldsymbol{T}}{T_{s}},
$$

where $I$ is the total number of stimulus combinations (given by $\left(\begin{array}{l}8 \\ 2\end{array}\right)=28$ for eight reproduction methods); $f_{n_{i}}$ is the frequency of use of attribute $n$ for the $i$ th combination; $p_{i}$ is the mean absolute preference score (scaled as detailed in Section III-A) for the $i$ th combination; $\boldsymbol{T}$ is a vector containing the total number of responses from each participant group (trained and untrained); and $T_{s}$ is the value of $\boldsymbol{T}$ for the participant group $s$ that produced the $n$th attribute. The term $\frac{\max T}{T_{s}}$ serves to normalise the attribute score to remove the effect of the total number of responses given by a particular participant group, by considering the proportion of total responses rather than an absolute frequency.

Figure 2 shows the attribute scores for all attributes. There is a knee-point in the curve after the first seven attributes, which are enveloping, horizontal width, output quality, surrounding, amount of distortion, overall spectral balance, and immersion. Four of these attributes appear to relate to the sensation of envelopment; enveloping and immersion were considered to be identical by $90 \%$ of participants [8], and the definitions of these attributes-as well as surrounding and horizontal width-exhibit considerable overlap.

\section{B. Reproduction method comparison}

The frequency of attribute use can also be broken down by examining the stimulus pairs alongside which each attribute was elicited, in order to determine likely reasons for preference for one specific system over another. Without attribute rating data, it is not possible to definitively say whether there is a positive or negative correlation between a particular attribute and preference; however, it is possible to determine the attributes that were being used by participants when making comparisons between two methods. The frequency of attribute use can be visualised using histograms. However, plotting twenty-eight histograms - each with fifty-one attributes-is cumbersome and does not allow for a quick, easy interpretation of the data. Consequently, the data was visualised as shown in Figure 3. Each bar relates to a particular pair of reproduction methods, with one of the two methods in the pair shown in groups (separated by dashed vertical lines) and the second method along the x-axis. The bars show the percentage of responses accounted for by each attribute; the attributes are differentiated by colour. In order to clarify the visualisation, a threshold was

\footnotetext{
${ }^{1}$ Each attribute is derived from a number of listener responses by way of a clustering algorithm and group discussion. Of all the elicited responses contributing to a particular attribute, some might include the exact attribute name, whereas others might only include related terms. In this paper, 'frequency of attribute use' is used to mean the number of elicited responses that contributed to a particular attribute, regardless of whether or not each of those responses included the exact attribute name. The attribute derivation is further discussed in [6].
} 


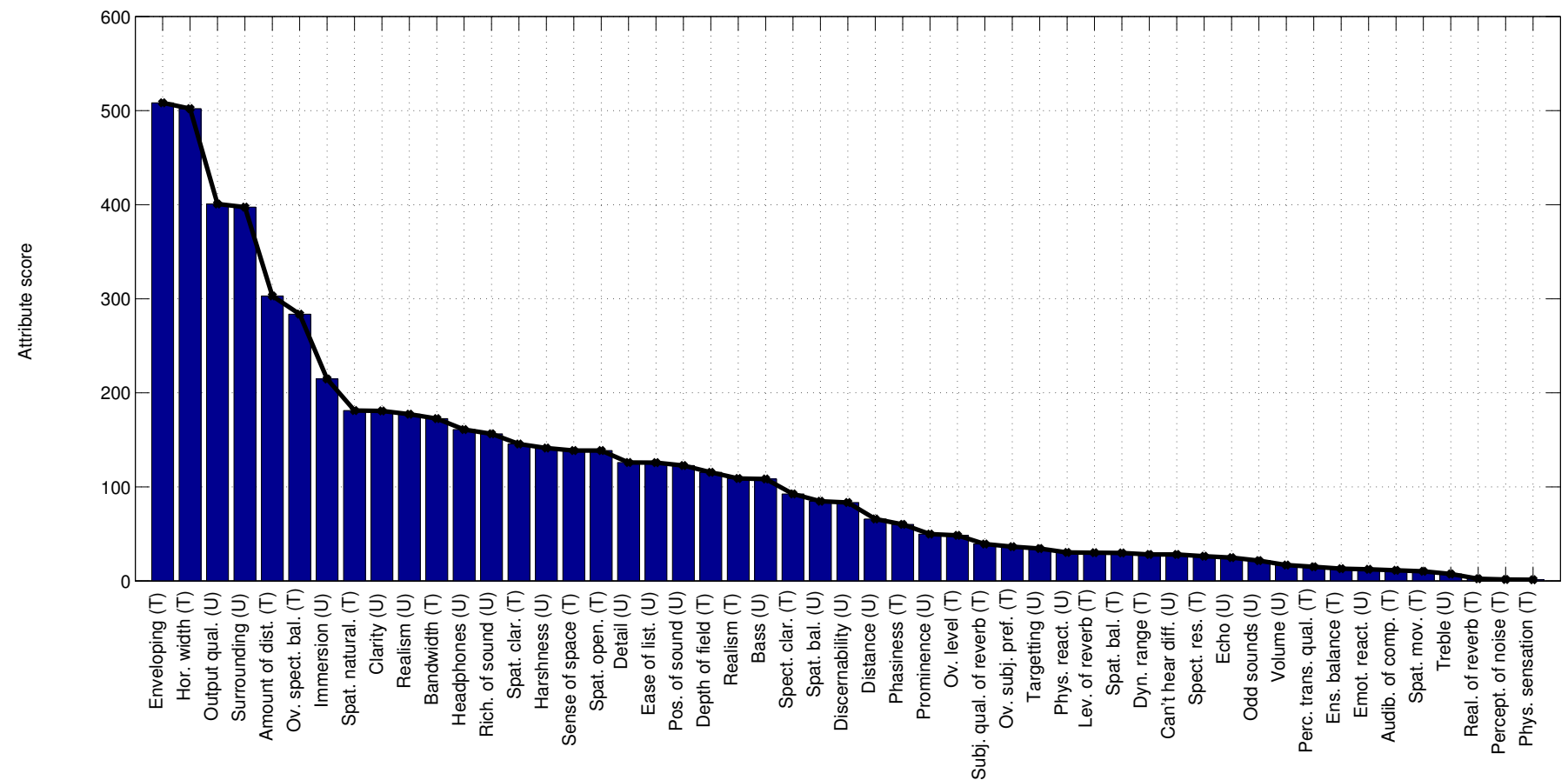

Fig. 2: Attribute score for all attributes. The participant group that elicited each attribute is indicated next to the label: (U) refers to the untrained listeners; (T) refers to the trained listeners.

set so that only attributes that account for more than $8 \%$ of the responses for each combination (selected manually to trade off showing as much information as possible against keeping the plot interpretable) are shown.

The plot can be read at three levels of detail: overall, for all comparisons involving a particular reproduction method, and by individual comparisons. The attribute enveloping features heavily across all comparisons, as does horizontal width. These attributes also had the highest attribute scores, suggesting that they are of particular importance and contribute greatly to listener preference. There are a number of attributes that stand out as being used alongside particular reproduction methods: headphones for the headphone reproduction; amount of distortion for the low-quality mono; surrounding for the mono, and spatial naturalness for the cuboid. Overall spectral balance features in the comparisons between the better-performing systems (e.g. stereo and 5-, 9-, and 22-channel), as well as the cuboid.

\section{INTERNAL PREFERENCE MAPPING}

Dimensional analysis is often used in the sensory sciences to determine how products relate to each other (by positioning them in a multidimensional space) and to determine the perceptual dimensions that assessors use to judge products [9]. Often, ratings are collected on multiple pertinent attributes; external preference mapping is then performed as a dimension reduction technique in order to determine redundancy in the attribute set and identify the relationship between products and attributes. In such analyses, the attribute scores are generally averaged across participants. However, internal preference mapping is an alternative procedure in which principal component analysis (PCA) is performed on the participants' preference rating data (a products $\times$ participants matrix) [10] The resulting components are known as "preference dimensions', and participants are plotted as vectors in the preference space. The preference dimensions are orthogonal and explain a decreasing amount of variance. The direction and length of each participant vector gives an indication of how that participant's preference judgement was made; the length of the vector is proportional to the amount of variance explained by the dimensions shown. The products (in this case, reproduction methods) can also be plotted in the preference space, revealing the direction and magnitude of preference for each product, and differences in preference exhibited by different listener groups. The distance between a participant and product in the space is proportional to the extent of that participant's preference for the product.

The paired comparison preference data was preprocessed to facilitate internal preference mapping (Section III-A); the preference mapping was then performed for the overall data (i.e. all participants; Section III-B), and separately for the trained and untrained listeners (Section III-C). Finally, the attributes discussed in Section II were used to label the resulting dimensions (Section III-D).

\section{A. Data preprocessing}

The internal preference mapping was performed by conducting PCA on a matrix of preference scores with participants in rows and reproduction methods in columns. The matrix was compiled as follows.

1) To account for individual differences in usage of the preference scale, the paired comparison preference 


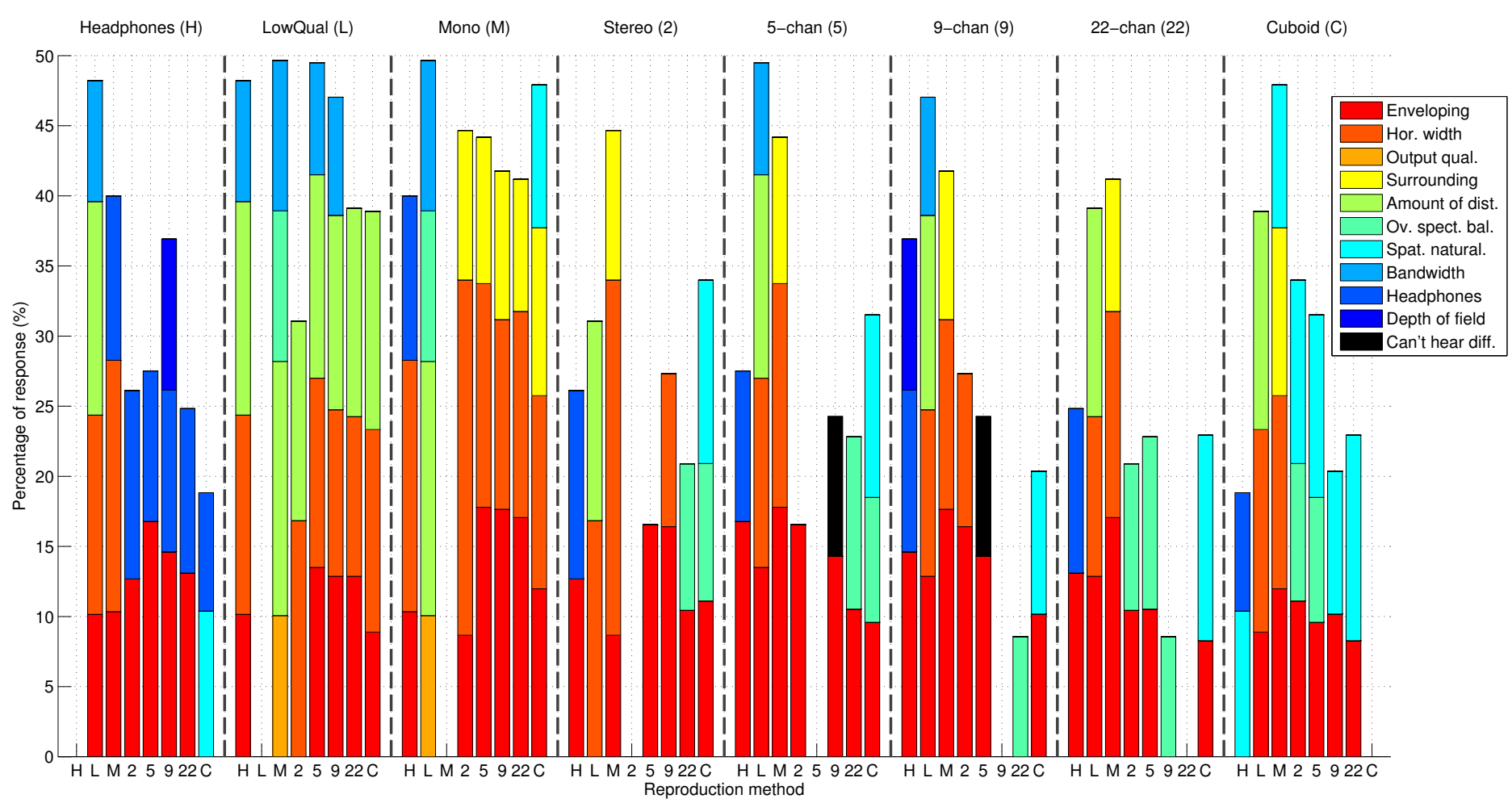

Fig. 3: Bar chart showing how attributes were used for comparisons between specific systems. The bar segments show the percentage of all responses for the given combination that were made up by a particular attribute (indicated by bar color). Only attributes that make up at least $8.0 \%$ of the responses for each combination are shown. The reproduction method short labels are outlined in the group titles at the top of the figure.

scores for each participant were scaled by dividing each score by the standard deviation of all scores for that participant.

2) For each participant, an 8-by-8 (i.e. the number of reproduction methods) preference matrix $P$ was created by summing (over all programme material items) the scaled preference score for each combination of reproduction methods. For example, if reproduction method 1 was preferred to reproduction method 2 with a score of $x, P_{1,2}$ would be increased by $x$, and $P_{2,1}$ would be decreased by $x$.

3) Single-figure preference scores for each reproduction method were produced by summing the rows of the preference matrix described in point two [11].

\section{B. All participants}

Principal component analysis was performed on the participants $\times$ reproduction methods matrix for all participants, and the percentage of variance explained by each resulting dimension was calculated. The first dimension accounted for $92.9 \%$ of the variance in preference scores, with a further $4.2 \%$ accounted for by the second dimension. The first two dimensions were therefore analysed by plotting a biplot showing participant preference vectors and reproduction systems (Figure 4).

The large amount of variance explained by the first dimension suggests that it has a strong bearing on overall preference

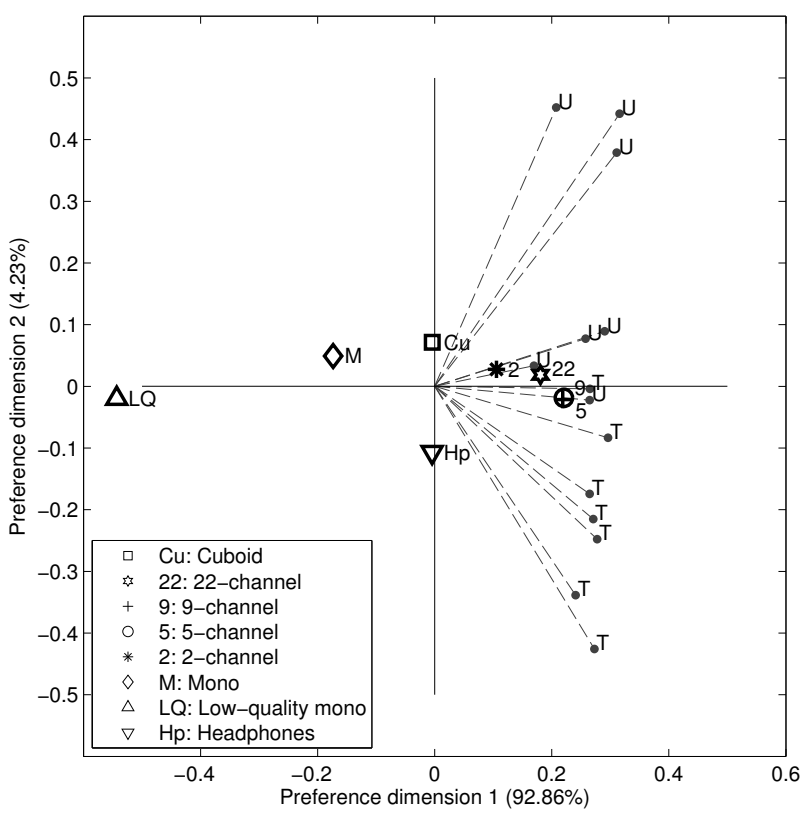

Fig. 4: Internal preference mapping biplot. Participant preference vectors are shown by dashed lines. The participant groups are indicated by ' $U$ ' for untrained listeners and ' $T$ ' for trained listeners. 
and is unlikely to relate to a single perceptual attribute. The low-quality mono reproduction method has a strong negative position on the first dimension. The remaining systems are clustered together: the mono reproduction is the next least preferred; the cuboid and headphones are approximately equally positioned on dimension one; they are followed by the stereo then 22-channel systems; and finally, the 9- and 5-channel systems are most preferred and have almost equal positions in the space. The participant preference vectors show that all participants agree in preference along the first dimension (i.e., they all have positive positions on dimension one). However, the preference vectors are separated on the second dimension: the majority of trained listeners have negative positions on dimension two, whilst the untrained listeners have positive positions. The positions of the reproduction methods on the second dimension suggest that this is due to a difference in preference for the headphone reproduction; the trained listeners show a greater preference for headphone reproduction than the untrained listeners.

The results described above are likely to be highly influenced by the apparent strong dislike for the low-quality mono and mono reproduction; therefore, these reproduction methods were removed from the data (before point two of the preprocessing described in Section III-A) and the analysis repeated. The majority of the variance $(71.6 \%)$ is still explained by the first dimension; however, the proportion of variance accounted for by the second dimension increases to $22.2 \%$, and the third dimension to $4.4 \%$. The biplots for dimensions one to three are shown in Figure 5. Figure 5a emphasises the differentiation between the cuboid and headphone reproduction methods, with the former generally preferred by untrained listeners and the latter by trained listeners. Figure $5 b$ shows that there is a wide spread of preference vector positions on dimension three; this suggests that there are no consistent trends. The stereo reproduction method has a moderate negative position on this dimension; the other methods fall close to 0 .

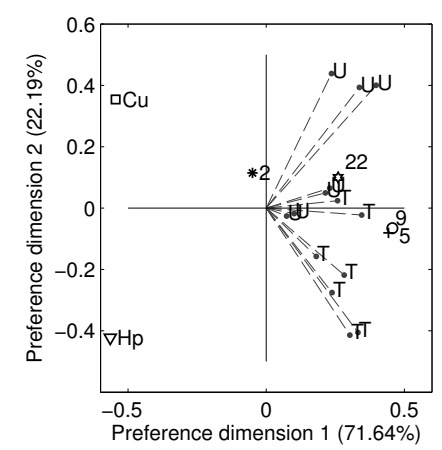

(a) Dimensions 1 and 2

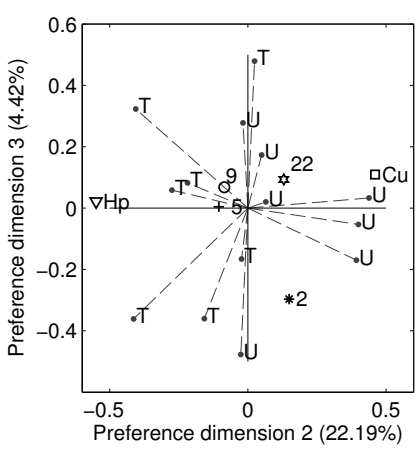

(b) Dimensions 2 and 3
Fig. 5: Internal preference mapping biplots for dimensions $1-3$, with mono and low-quality mono reproduction methods removed. Participant preference vectors are shown by dashed lines. The participant groups are indicated by ' $U$ ' for untrained listeners and ' $\mathrm{T}$ ' for trained listeners. The reproduction method labels and markers are detailed in the legend in Figure 4.

\section{Listener groups}

The results presented above highlight a difference in preference between the trained and untrained listener groups. Therefore, the analysis was performed separately for each group of listeners. Biplots are shown in Figure 6. As for the overall results, the vast majority of variance is explained by the first preference dimension $(95.2 \%$ and $96.3 \%$ for the untrained and trained listeners respectively). Differences can be seen in the positioning of the reproduction methods between the two groups; the untrained listeners have approximately equal preference for the mono and headphone reproduction methods, whilst the trained listeners have a higher preference for the headphone method and a lower preference for the ambisonic cuboid than the untrained listeners. Another difference is seen in the participant groupings. The majority of the trained listeners are in strong agreement along dimension one, with only two outlying participants who are positioned in opposite directions on dimension two. The untrained listeners have a much greater spread, particularly in the second dimension.

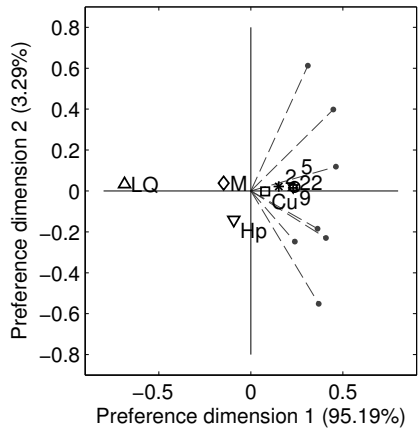

(a) Untrained listeners

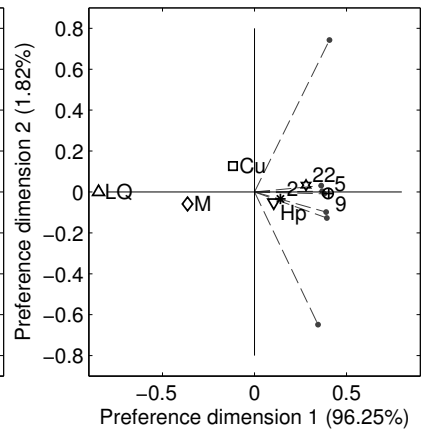

(b) Trained listeners
Fig. 6: Internal preference mapping biplots (dimensions 12) for untrained and trained listeners. Participant preference vectors are shown by dashed lines. The reproduction method labels and markers are detailed in the legend in Figure 4.

\section{Labelling the dimensions}

Without ratings on all of the attributes, it is not possible to completely accurately position the attributes in the dimensional perceptual space. However, the analysis in Section II can be used to help interpret the preference dimensions produced in the internal preference mapping. The biplots show the relative positions of reproduction systems in the dimensional space; by taking two systems that are at opposite ends of one preference dimension (especially if they are similarly positioned in other dimensions), and using Figure 3 to see what attributes were used to differentiate between those systems, the dimensions can be approximately labelled. This approach is similar to the method used by Hall [12], who asked participants to describe the attribute that was changing when they were presented with stimuli that had monotonically-varying positions on a particular dimension.

As discussed in Section III-B, Figure 4 shows that the majority of the variance in the data is explained by the first preference dimension; consequently, it is highly likely that multiple attributes are related to this dimension. Enveloping and horizontal width were shown to be used consistently for all conditions and to have high perceptual importance based on the 
attribute score. The low-quality mono had an extreme negative position on preference dimension one; amount of distortion was frequently used alongside comparisons featuring the lowquality mono reproduction method, and is therefore also likely to contribute to this dimension.

In Figure 5a, the cuboid and headphone reproduction methods are separated on the second preference dimension, which accounts for an increased proportion of variance when the low-quality mono and mono methods were removed from the analysis. Figure 3 shows two attributes that were used alongside a relatively high percentage of comparisons involving these two methods: headphones (untrained listeners) and spatial naturalness (trained listeners). The second dimension can therefore be considered to relate to these two attributes: for the untrained listeners, whether or not they were wearing headphones greatly influenced their ratings (and headphonewearing seemed to give reduced preference based on the direction of the participant preference vectors); for the trained listeners, the difference in spatial naturalness between the cuboid and other methods was important. This is perhaps unsurprising given that first-order ambisonic recording and decoding was used, which can have a small sweet spot and undesirable phase effects resulting in inconsistent localisation [13]. Finally, Figure 5b shows that the stereo method is separated from the others on the third preference dimension. This dimension only accounts for a very small amount of variance, and it is difficult to label. Figure 3 shows that overall spectral balance was used for comparisons involving stereo and the high channel-count reproduction methods; it is possible that the third dimension is related to this attribute.

\section{CONCLUSIONS}

There are many spatial audio reproduction systems available, and it is therefore desirable to understand the factors that contribute to quality of listener experience, in order that future systems can be perceptually optimised. In the work reported above, the results of a listener preference test for different spatial audio reproduction methods were analysed. Three research aims were stated in Section I; the principal findings of the work that address these aims are stated below.

The perceptual importance of each member of a set of attributes, elicited alongside preference ratings, can be objectively determined using a metric (Equation 1) that takes into account: (i) the frequency of use of attributes; and (ii) the magnitudes of the preference judgements alongside which these attributes are used. Using experimental data comparing audio reproduction systems, this metric indicates that seven attributes are particularly important: enveloping, horizontal width, output quality, surrounding, amount of distortion, overall spectral balance, and immersion.

Internal preference mapping can be used to position participant preference vectors and reproduction method scores in a dimensional space. For the experiment conducted, this space shows that: (i) nearly all of the variance in preference is accounted for by a single dimension, relating to envelopment, horizontal width, and amount of distortion; and (ii) in the absence of distorted and mono reproduction methods, a second dimension-relating to spatial naturalness and the wearing of headphones-becomes more important. The pronounced negative position of a low-quality loudspeaker reaffirms the importance of high timbral quality [14] to listener experience (as emphasised by the importance of amount of distortion). Spatial factors are also important (as seen through extensive use of the attribute enveloping for nearly all combinations).

Trained listeners exhibit a greater preference for headphone reproduction than untrained listeners, but lower preference for ambisonic reproduction. Trained listeners also show greater inter-participant agreement.

\section{A. Outlook and future work}

Knowledge of the important perceptual attributes of spatial audio replay can help to maximise quality of listening experience; future research should focus on developing meters of the important attributes as well as designing systems that are intended to preserve or optimise these attributes. Objectbased audio provides an opportunity to repurpose content for different reproduction systems regardless of the available loudspeaker channels. An interesting direction for future research would be to investigate high channel-count surround sound systems using low-quality loudspeakers; this might enable a dimensional analysis to more clearly reveal the reasons for listener preference.

\section{REFERENCES}

[1] F. Rumsey, "Subjective assessment of the spatial attributes of reproduced sound," in Proceedings of the 15th Audio Engineering Society International Conference on Audio, Acoustics, \& Small Spaces (Paper No. 15-012), Oct. 1998.

[2] ITU-R, "ITU-R Recommendation BS.775-3: Multichannel stereophonic sound system with and without accompanying picture," 2012.

[3] — , "ITU-R Recommendation BS.2051-0: Advanced sound system for programme production," 2014.

[4] S. Füg, A. Hölzer, C. Borß, C. Ertel, M. Kratschmer, and J. Plogsties, "Design, coding and processing of metadata for object-based interactive audio," in AES 137th Convention, Los Angeles, USA, 2014.

[5] S. Spors, H. Wierstorf, A. Raake, F. Melchior, M. Frank, and F. Zotter, "Spatial Sound With Loudspeakers and Its Perception: A Review of the Current State," Proceedings of the IEEE, vol. 101, no. 9, pp. 1920-1938, Sep. 2013.

[6] J. Francombe, T. Brookes, R. Mason, and J. Woodcock, "Analysis of listener preference for spatial audio reproduction methods," unpublished.

[7] J. Francombe, T. Brookes, R. Mason, and F. Melchior, "Loudness matching multichannel audio program material with listeners and predictive models," in AES 139th Convention, New York, USA. Audio Engineering Society, Oct. 2015.

[8] J. Francombe, T. Brookes, and R. Mason, "Identification of the perceptual differences between spatial audio reproduction methods," unpublished.

[9] H. T. Lawless and H. Heymann, Sensory evaluation of food: principles and practices. New York: Springer, 1999.

[10] K. Greenhoff and H. J. H. MacFie, "Preference mapping in practice," in Measurement of Food Preferences, H. J. H. MacFie and D. M. H. Thomson, Eds. New York: Springer, 1994, pp. 137-166.

[11] E. Parizet, N. Hamzaoui, and G. Sabatié, "Comparison of some listening test methods: a case study," Acta Acustica united with Acustica, vol. 91, pp. 356-364, 2005.

[12] J. L. Hall, "Application of multidimensional scaling to subjective evaluation of coded speech," The Journal of the Acoustical Society of America, vol. 110, no. 4, pp. 2167-2182, 2001.

[13] F. Rumsey, Spatial audio. Oxford: Focal Press, 2001

[14] F. Rumsey, S. Zieliński, R. Kassier, and S. Bech, "On the relative importance of spatial and timbral fidelities in judgments of degraded multichannel audio quality," The Journal of the Acoustical Society of America, vol. 118, no. 2, pp. 968-976, 2005. 